Urology

Elsevier Editorial System(tm) for European

Manuscript Draft

Manuscript Number: EURUROL-D-16-01486R1

Title: Cytoreductive nephrectomy in the tyrosine kinase inhibitor era: a question that may never be answered

Article Type: Editorial

Keywords: renal cell cancer; clinical trials; cytoreductive; nephrectomy; tyrosine kinase inhibitors; sunitinib; immunotherapy

Corresponding Author: Dr. Grant Duncan Stewart, BSC (Hons), MBChB, ERCSEd, PhD

Corresponding Author's Institution: Cambridge University

First Author: Grant Duncan Stewart, BSC (Hons), MBChB, FRCSEd, PhD

Order of Authors: Grant Duncan Stewart, BSC (Hons), MBChB, FRCSEd, PhD;

Michael Aitchison; Axel Bex; James Larkin; Claire Lawless; Arnaud Mejean;

Paul Nathan; Grenville Oades; Jean-Jacques Patard; James Paul; Alain

Ravaud; Bernard Escudier 
Cytoreductive nephrectomy in the tyrosine kinase inhibitor era: a question that may never be answered

Conflicts of interest:

A Ravaud and A Mejean are co-Cls of CARMENA

M Aitchison was UK lead investigator for CARMENA

A Bex is co-Cl for SURTIME

None of the other authors have conflicts of interest 


\section{Cytoreductive Nephrectomy in the Tyrosine Kinase Inhibitor Era: A Question That May Never Be Answered}

G.D. Stewart, M. Aitchison, A. Bex, J. Larkin, C. Lawless, A. Méjean, P. Nathan, G. Oades, J.-J. Patard, J. Paul, A. Ravaud, B. Escudier, on behalf of the Renal Cross Channel Group

Despite great interest, two randomised controlled trials (RCTs) of cytoreductive nephrectomy in the tyrosine kinase inhibitor setting in metastatic renal cell carcinoma have either closed early (SURTIME) or are recruiting very slowly (CARMENA) after 7 yr. Challenges in RCT delivery in uro-oncologic surgery are many. Multiple steps are needed to ensure strong recruitment to trials addressing important urologic cancer questions. Feasibility/pilot studies are key stepping stones towards successful delivery of surgical RCTs. 


\section{Cytoreductive Nephrectomy in the Tyrosine Kinase Inhibitor Era: A Question That May Never Be Answered}

Grant D. Stewart ${ }^{\mathrm{a}, *}$, Michael Aitchison ${ }^{\mathrm{b}}$, Axel Bex ${ }^{\mathrm{c}}$, James Larkin ${ }^{\mathrm{d}}$, Claire Lawless ${ }^{\mathrm{e}}$, Arnaud Méjean ${ }^{\mathrm{f}}$, Paul Nathan ${ }^{\mathrm{g}}$, Grenville Oades ${ }^{\mathrm{h}}$, Jean-Jacques Patard ${ }^{\mathrm{i}}$, James Paul ${ }^{\mathrm{e}}$, Alain Ravaud $^{\mathrm{j}}$, Bernard Escudier ${ }^{\mathrm{k}}$, on behalf of the Renal Cross Channel Group

a Academic Urology Group, University of Cambridge, Addenbrooke's Hospital, Cambridge, UK

${ }^{\mathrm{b}}$ Royal Free Hospital, London, UK

${ }^{\mathrm{c}}$ Netherlands Cancer Institute, Amsterdam, Netherlands

${ }^{\mathrm{d}}$ Royal Marsden Hospital, London, UK

${ }^{\text {e }}$ CRUK Clinical Trials Unit, The Beatson West of Scotland Cancer Centre, Glasgow, UK

${ }^{\mathrm{f}}$ Université Paris Descartes, Paris, France

${ }^{g}$ Mount Vernon Hospital, Northwood, UK

${ }^{\text {h }}$ Queen Elizabeth University Hospital, Glasgow, UK

${ }^{\mathrm{i}}$ Hôpital Bicetre, Paris, France

${ }^{\mathrm{j}} \mathrm{CHU}$ Bordeaux, Bordeaux, France

${ }^{\mathrm{k}}$ Gustave Roussy Institute, Paris, France

* Corresponding author. Academic Urology Group, University of Cambridge, Box 43, Addenbrooke's Hospital, Cambridge Biomedical Campus, Hill's Road, Cambridge CB2 0QQ, UK. Tel. +44 1223256211.

E-mail address: gds35@cam.ac.uk (G. Stewart).

Renal cancer surgeons are acutely aware of pre-eminent data from the immunotherapy era demonstrating a significant survival advantage for patients with metastatic renal cell cancer (mRCC) having cytoreductive nephrectomy $(\mathrm{CNx})$ before IFN- $\alpha$ treatment $[1,2]$. However, oncologic treatments given to patients with $\mathrm{mRCC}$ have radically changed in the current era in which pan-tyrosine kinase inhibitors (TKIs) and those specifically targeting VEGF or mTOR are used. In 2009/2010 the urological surgery community widely supported the launch of two randomised controlled trials that aimed to assess the place of $\mathrm{CNx}$ among mRCC patients treated with TKIs, as well as the timing of CNx in relation to TKI administration. In the French CARMENA trial (NCT00930033), patients were to be randomised to CNx and sunitinib versus sunitinib alone without CNx (Supplementary Fig. 1). In the EORTC sponsored SURTIME trial (EORTC 30073; NCT01099423), the sequencing of drug and surgical therapies was to be assessed. Patients were randomised to sunitinib followed by $\mathrm{CNx}$ and subsequent sunitinib versus CNx followed by sunitinib (Supplementary Fig. 2). However, recruitment to these two studies has been hugely challenging.

After initial robust recruitment in France, CARMENA was opened to recruitment in the UK in May 2011. A total of 26 sites around the UK were opened for CARMENA recruitment. However, CARMENA was closed to recruitment in the UK in 2014, as only 14 patients were recruited over 4 yr. However, the CARMENA study continues to recruit slowly in France (411 of 576 patients recruited) and it is likely to complete recruitment in September 2017; the study is estimated to end $6 \mathrm{yr}$ later than originally planned. In an attempt to try and determine why this study failed to recruit in a nation with a robust trial infrastructure, an investigator questionnaire was sent to the UK investigators. Responses indicated that there was a lack of patient and clinician equipoise and an inability of the clinical team to convince patients to be 
randomised (Table 1). Within the investigator questionnaire, 34 varied $\mathrm{mRCC}$ clinical scenarios were described, and investigators were asked if they would recommend surgery, drug treatment, best supportive care, or entry into CARMENA. Of the 17 respondents $(65 \%$ response rate), the five urologists stated that their preferred management strategy would be CARMENA for a median of 20 of the scenarios (range 11-22), whereas the 12 oncologists opted for CARMENA for a median of 8.5 scenarios (range 6-19). Thus, urologists appeared to have greater levels of equipoise for the study. However, if there is one key individual within the clinical team who lacks equipoise, this is usually transferred to the patient, making recruitment more challenging.

The SURTIME study has also been hugely challenging, with poor recruitment in many centres. Efforts were made by the European Organisation for Research and Treatment of Cancer (EORTC) to improve accrual via online education tools and regular updates. Accrual was strongest in the Netherlands and Canada, and best in centres with a main focus on RCC management, where study eligibility was discussed at multidisciplinary tumour teams with urologists and oncologists together. However, SURTIME eligibility criteria were complex and were considered among the main reasons for the poor accrual. This was especially true for smaller centres, for which small numbers of patients precluded experience with the entry criteria. In addition, although surgery and therapy were offered in both arms, it proved difficult to convince patients assent to randomisation. The study closed early in 2016 and is probably underpowered to show differences in the primary and secondary endpoints of progression-free survival and overall survival, but may answer the question of rapid progression after pretreatment and interruption for surgery.

Therefore, the main hope for level 1 evidence regarding the place of $\mathrm{CNx}$ in $\mathrm{mRCC}$ in the TKI era comes from recruitment to CARMENA study by the French team. However, there are some concerns that this study will only answer the question of whether both arms are "equivalent"; recruitment of 1134 patients is needed to be able to determine if either arm was deleterious. Thus, it may be that lower levels of evidence suggesting that $\mathrm{CNx}$ is beneficial in selected situations such as patients predicted to have life expectancy of $>1 \mathrm{yr}$ are the best we will have to answer this common clinical dilemma [3]. It is of concern that there is evidence that $\mathrm{CNx}$ is now underutilised, especially in non-academic centres and for Black and uninsured patients. This underutilisation of $\mathrm{CNx}$ was associated with 10-mo poorer $\mathrm{mRCC}$ survival [4].

Recruitment issues in surgical trials are not specific to urology. It has been recognised for a number of years that randomised controlled trials (RCTs) in surgery are exceedingly challenging for a number of reasons $[5,6]$ : surgeon and patient equipoise; perceived threats to the surgeon's personal interests; lack of funding, infrastructure, and experience in data collection; operative learning curves; and blinding. A recent study revealed that one in five surgical RCTs were stopped early, and one in three completed trials did not publish after a median of $4.9 \mathrm{yr}$ [7]. The commonest reason for discontinuation was, as in the SURTIME example, poor recruitment. Numerous initiatives to improve recruitment to surgical RCTs have been attempted and are currently ongoing to improve renal cancer surgery trials, but it is clear that there is no single solution that will improve the situation (Table 2). Thus, in addition to the multiple sensible measures to improve recruitment once a trial has opened, it is now recommended that for any renal cancer surgery-related RCT, a feasibility or pilot study in a limited number of patients and centres be instigated before launching the main trial. Results from successful feasibility/pilot studies will allow the launch of a fully powered study, may influence the power calculation for the full study, and could provide a cadre of engaged urologists to deliver future clinical trials.

There are signs that in surgery in general the tide is changing in terms of delivery of successful RCTs [8]. Despite this, as we move into the second generation of the RCC 
immunotherapy, it is likely that we will never answer the question of the place of $\mathrm{CNx}$ in patients treated with TKIs.

Conflicts of interest: Alain Ravaud and Arnaud Méjean are co-Chief Investigators of CARMENA. Michael Aitchison was the UK lead investigator for CARMENA. Axel Bex is the co-Chief Investigator for SURTIME. The remaining other authors have nothing to disclose.t

Acknowledgments: CARMENA is sponsored by Assistance Publique-Hôpitaux de Paris (APHP). CARMENA-UK was funded by Cancer Research UK and administered by the CRUK Clinical Trials Unit, The Beatson West of Scotland Cancer Centre, Glasgow. SURTIME was sponsored by the European Organisation for Research and Treatment of Cancer (EORTC).

\section{References}

[1] Flanigan RC, Salmon SE, Blumenstein BA, et al. Nephrectomy followed by interferon alfa-2b compared with interferon alfa- $2 b$ alone for metastatic renal-cell cancer. $N$ Engl J Med 2001;345:1655-9.

[2] Mickisch GH, Garin A, van Poppel H, de Prijck L, Sylvester R, European Organisation for Research and Treatment of Cancer (EORTC) Genitourinary Group. Radical nephrectomy plus interferon-alfa-based immunotherapy compared with interferon alfa alone in metastatic renal-cell carcinoma: a randomised trial. Lancet 2001;358:966-70.

[3] Heng DYC, Wells JC, Rini BI, et al. Cytoreductive nephrectomy in patients with synchronous metastases from renal cell carcinoma: results from the International Metastatic Renal Cell Carcinoma Database Consortium. Eur Urol 2014;66:704-10.

[4] Hanna N, Sun M, Meyer CP, et al. Survival analyses of metastatic renal cancer patients treated with targeted therapy with or without cytoreductive nephrectomy: a National Cancer Data Base study. J Clin Oncol. In press. http://dx.doi.org/10.1200/JCO.2016.66.7931

[5] Horton R. Surgical research or comic opera: questions, but few answers. Lancet 1996;347:984-5.

[6] McCulloch P, Taylor I, Sasako M, Lovett B, Griffin D. Randomised trials in surgery: problems and possible solutions. Br Med J 2002;324:1448-51.

[7] Chapman SJ, Shelton B, Mahmood H, Fitzgerald JE, Harrison EM, Bhangu A. Discontinuation and non-publication of surgical randomised controlled trials: observational study. Br Med J 2014;349:g6870.

[8] Editorial. The struggle for better research in surgery. Lancet 2016;387:1970. 
Table 1 - Selection of quotes in response to the investigator questionnaire that illustrate the lack of clinician and patient equipoise

"Randomisation is difficult and if offered surgery as a possible treatment, most patients decided to have it off trial."

"Relatively few patients with clinical equipoise."

"Patient choice was our main failure."

"Patients unwilling to be randomised between surgical and nonsurgical option. Patients often have strong views as to whether they would want to undergo surgery or not in a palliative setting."

"There was rarely equipoise at MDT discussion"

"Unwillingness to recruit due to surgeon/oncology bias."

"Many patients I saw either 'obviously' needed a nephrectomy or 'obviously' needed oncology. I did not want to delay their treatment."

MDT $=$ multidisciplinary team .

Table 2 - Recommendations for future surgery-related randomised controlled trials

Canvassing of the speciality regarding key questions for clinical trials (eg, using Delphi process)

Iterative discussion process with NCRI Clinical Studies Group during development

Initial pilot or feasibility study (refine recruitment procedures and inform recruiter training by piloting recruitment materials and determining reason for screening failures)

Consider a clinical nurse specialist to provide information in an unbiased manner with enough time for full discussion

Confirm commitment and explicitly make the case for equipoise with potential investigators at each site via an interview process

Education and training programme for recruiters

Ensure a clear reward process (authorship rights, research nurse funding) for high recruiters 


\section{Supplementary material}

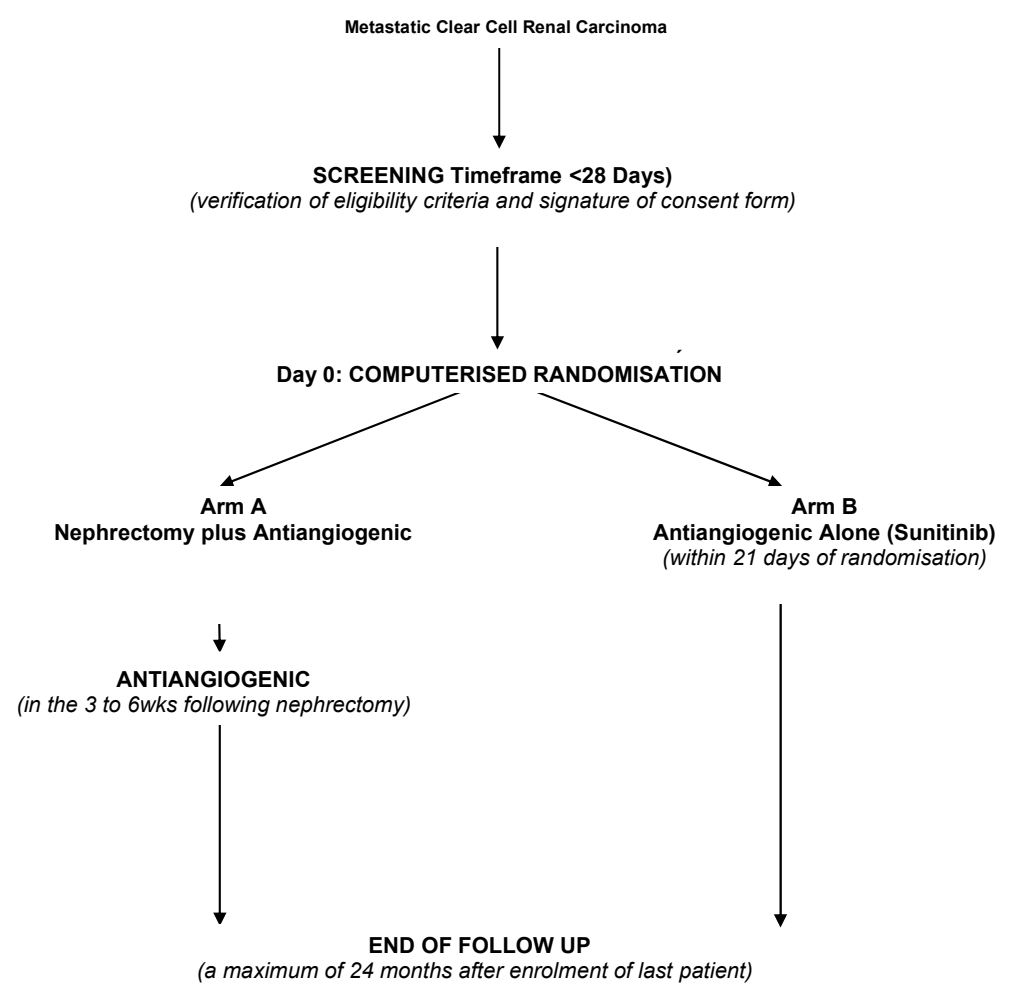

Supplementary Fig. 1 - Schema of the CARMENA trial.

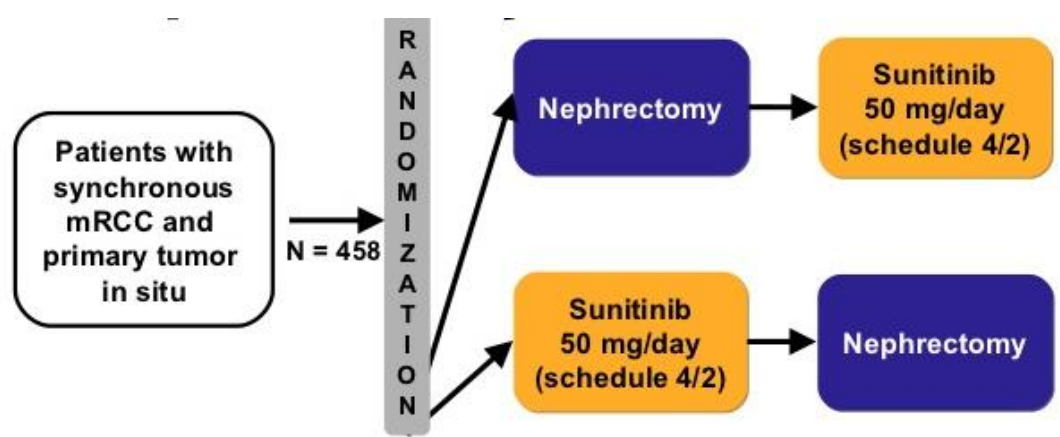

* Primary end point: PFS

* Secondary end points: OS, association with prognostic gene and protein expression profiles

Supplementary Fig. 2 - Schema of the SURTIME trial. $\mathrm{mRCC}=$ metastatic renal cell carcinoma; $\mathrm{PFS}=$ progression-free survival; $\mathrm{OS}$ = overall survival. 


\section{EURUROL-D-16-01486}

Title: Cytoreductive nephrectomy in the tyrosine kinase inhibitor era: a question that may never be answered

\section{Revision notes (our response to reviewers' comments in italics)}

Reviewer \#1: Well written, informative, with insider information about the status of these highly anticipated trials.

Thank you

Reviewer \#2: Excellent piece by prominent key opinion leaders in RCC and clinical trials about CARMEN and SURTIME.

It is unfortunate that important RCTs are not accruing as well. Many reasons were cited, all valid. Would suggest to have references for each one (e.g. surgeon and patient equipoise, personal prestige, lack of funding, infrastructure and experience in data collection, operative learning curves, and blinding). I also suggest adding role of academic/cooperative groups vs. industry trials. Will accrual and finishing trials be more successful with industrysurgical trials?. Would also suggest to expand on "personal prestige" as this is not a specific term. Perhaps the authors meant "personal agenda, personal opinion, personal interest, etc..."

Thank you.

The potential reasons for difficulties in recruiting to surgical trials are described in the referenced BMJ paper (McCulloch P, Taylor I, Sasako M, Lovett B, Griffin D. Randomised trials in surgery: problems and possible solutions. BMJ 2002;324:1448-51). This reference is given on line 86.

We have not see any difference in trial accrual and completion based on being an academic or industrial study, the completed adjuvant studies ASSURE (academic studies: ECOG/ACRIN) and S-TRAC (industrial study: Pfizer) are examples of this assumption. Co-operation between urologist and oncologist is the most important factor and not if lead by industry or academic institution. As this is not something we can robustly evidence, we thank the reviewer but would prefer not to include this discussion in the manuscript.

We have altered the confusing term 'personal prestige' to a more descriptive term 'perceived threat to surgeon's personal interests' on line 87.

Reviewer \#3: Well-written, very interesting including from a surgeon's standpoint.

Thank you 
Reviewer \#4: Well done editorial piece that summarizes the status of cytoreductive surgery in the era of targeted therapy. Could use a bit of editing for better English.

Thank you

Reviewer \#5: Nicely put together editorial. Two minor suggestions:

-No line numbers are provided on the submission, in accordance with European Urology guidelines

-In the first sentence, the use of "IL-2" is somewhat confusing, as the randomized trials the authors are referencing were conducted among patients treated with IFN-alpha.

Continuous line numbers have been inserted

Thank you for identifying the error regarding intermixing of IL-2 and IFN-a. We have removed reference to IL-2 and restructured sentence 1 in lines 25-28. 